\title{
Transmitterfunktionen in der menschlichen Epidermis am Beispiel keratinozytärer Glutamat- rezeptoren*
}

\section{Transmitter Functions in Human Epidermis, Using Keratinocytic Glutamate Receptors as an Example}

Autor

Institut

\section{Fischer}

Universitätsklinik und Poliklinik für Dermatologie und Venerologie, Martin-Luther-Universität Halle-Wittenberg, Halle (Saale)

\section{Bibliografie}

DOI $10.1055 / \mathrm{s}-2007-967009$

Akt Dermatol 2007; 33 :

410-412 @ Georg Thieme

Verlag KG Stuttgart · New York ISSN 0340-2541

Korrespondenzadresse Priv.-Doz. Dr. Matthias Fischer HELIOS-Klinikum Aue Klinik für Dermatologie und Venerologie Gartenstr. 6 08280 Aue matthias.fischer@helioskliniken.de

\section{Zusammenfassung \\ $\nabla$}

In der Epidermis des Menschen werden zunehmend Transmitter und Rezeptoren gefunden, die ursprünglich ausschließlich im Nervensystem vermutet wurden. Hierzu zählen unter anderem Glutamat-, Acetylcholin- und Purinrezeptoren. Die einzelnen Rezeptorfamilien beeinflussen da-

In der Epidermis unterliegt die Regulation der Proliferation und Differenzierung des Keratinozyten komplexen, bis heute noch nicht vollständig geklärten Mechanismen. In den letzten Jahren wurden dabei zunehmend Transmitter-vermittelte Signalwege beschrieben, bei denen ursprünglich neurogene Botenstoffe über die Bindung an spezifischen Rezeptoren Einfluss auf die Funktion von Keratinozyten nehmen [1 - 3]. Ihrer chemischen Struktur und Eigenschaften nach können Transmitter in biogene Amine, Neuropeptide, Nukleotide, Aminosäuren und lösliche Gase unterteilt werden und binden meist an spezifischen Rezeptoren. Die hierdurch ausgelösten Signale sind vielgestaltig und können aus der Öffnung transmembranöser Ionenkanäle, Entleerung intrazellulärer Kalziumspeicher oder der Ausschüttung von Zytokinen bestehen [3-5]. Als Beispiel für eine ursprünglich neurogene Transmitter-/Rezeptorwirkung soll nachfolgend der aktuelle Kenntnisstand keratinozytärer Glutamatrezeptoren beschrieben werden.

L-Glutamat ist der wichtigste exzitatorische Transmitter im Nervensystem der Vertebraten, der seine Wirkung über die Bindung an verschiedenen ionotropen und metabotropen Glutamatrezeptoren entfaltet ( $\bullet$ Abb. 1). Metabotrope Glutamatrezeptoren erhöhen als G-Protein-gekoppelte Rezeptoren entweder über Gq-Phos-

\footnotetext{
* Vortrag beim wissenschaftlichen Jahres-Symposium der Berliner Stiftung für Dermatologie, 12. Mai 2007, Berlin.
}

bei sowohl die Proliferation und Differenzierung des Keratinozyten als auch die Barrierefunktion der Epidermis. Am Beispiel keratinozytärer Glutamatrezeptoren werden Transmitterfunktionen in der Epidermis beschrieben, die ursprünglich exklusiv dem Nervensystem zugeschrieben wurden.

pholipase $\mathrm{C}$ die intrazelluläre Calciumkonzentration (Rezeptoren der Gruppe I) oder führen über Gi zu einer Verminderung von intrazellulärem cAMP (Rezeptoren der Gruppen II + III) [6, 7]. Die ionotropen Glutamatrezeptoren hingegen sind ligandengesteuerte, nicht-selektive, postsynaptisch gelegene, transmembranöse Kationenkanäle [7]. Neben L-Glutamat sind noch eine Reihe spezifischer Agonisten bekannt, die selektiv an verschiedenen ionotropen Glutamatrezeptoren binden. Nach diesen selektiven Agonisten werden die ionotropen Glutamatrezeptoren weiter in AMPA- (Amino-3-hydroxy-5-methyl-4-isoxazolpropionat), Kainat- und NMDA-Rezeptoren (N-Methyl-D-Aspartat) unterteilt [7].

Die Existenz von Glutamatrezeptoren wurde bislang überwiegend im Nervensystem angenommen. In den letzten Jahren fanden sich jedoch vermehrt Hinweise auf eine Präsenz auch in nicht-neuronalen Zellen wie beispielsweise Keratinozyten [8-15]. Sowohl in der dermatologischen als auch der neurologisch/neuropathologischen Forschung ist der NMDA-Rezeptor der am besten untersuchte und charakterisierte Glutamatrezeptor. Bei ihm werden eine konstante R1-Komponente und variable R2- (NMDAR2A-D) und R3-Subtypen unterschieden [7], die für funktionelle Besonderheiten der jeweiligen Rezeptorsubtypen von besonderer Bedeutung sind. Keratinozyten exprimieren NMDA-Rezeptoren vom Typ NMDAR2D [10]. Über die R3-Kom- 


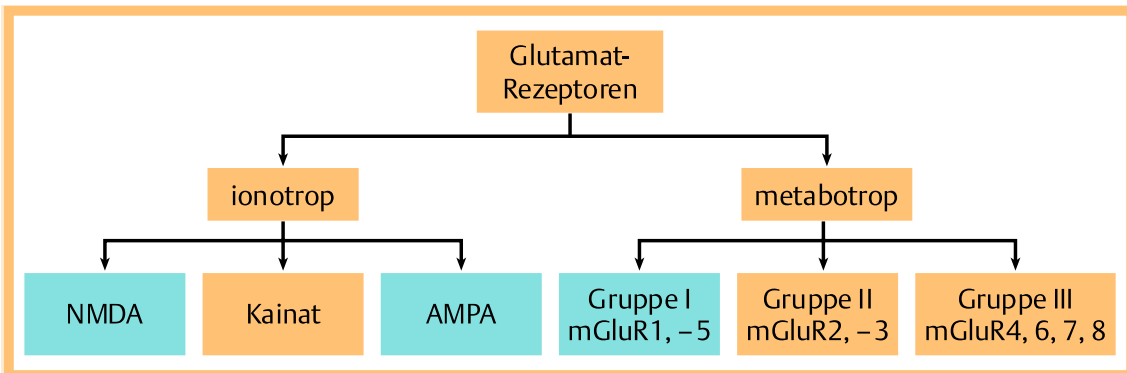

Abb. 1 Familie der Glutamatrezeptoren. Blau unterlegt: bislang bei Keratinozyten gefundene Subtypen.

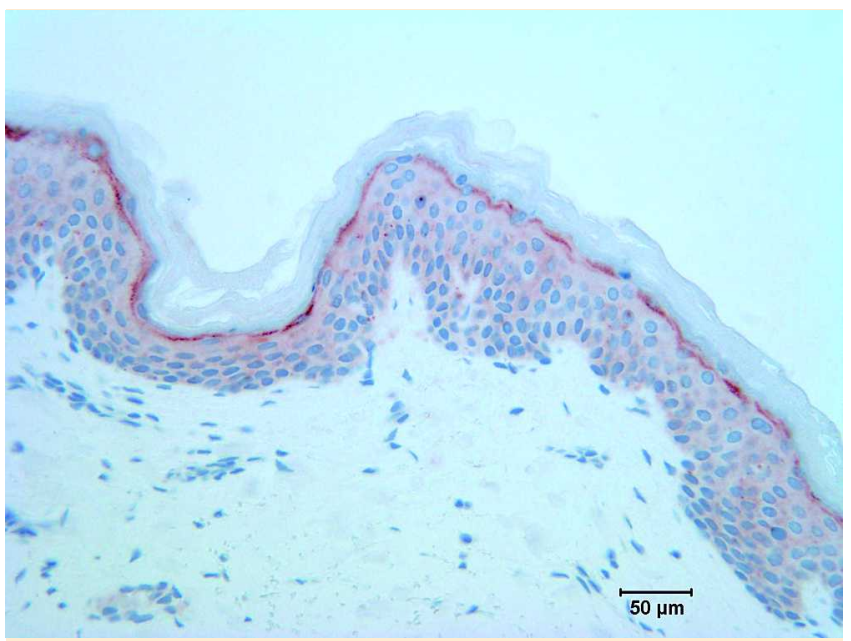

Abb. 2 Immunhistochemische Darstellung von Glutamatrezeptoren vom Typ des N-Methyl-D-Aspartat-Rezeptors in gesunder menschlicher Epidermis. Maximale Expression im Stratum granulosum, der intraepidermalen Kalziumkonzentration entsprechend.

ponente bei Keratinozyten liegen bislang keine Erkenntnisse vor. Innerhalb der Epidermis ist der NMDA-Rezeptor (NMDAR1) in der gesamten vitalen Epidermis nachweisbar, wobei sich eine besonders starke Ausprägung innerhalb des Stratum granulosum findet ( $\bullet$ Abb.2) $[8,9]$. Diese Verteilung stimmt sehr gut mit dem von Menon und Elias [16] beschriebenen Kalziumgradienten überein, der einen sprunghaften Anstieg innerhalb des Stratum granulosum zeigt. Dieser abrupte Kalziumanstieg wird als wichtiges Signal für die Differenzierung des Keratinozyten angesehen. Tatsächlich findet sich auch bei kultivierten Keratinozyten (HaCaT-Zellen, normale humane epidermale Keratinozyten (NHEK)) eine Erhöhung der intrazellulären Kalziumkonzentration nach Stimulation der Zellen mit NMDA [8]. Dieses Phänomen war durch die vorherige Blockade des Rezeptors mit dem NMDA-Rezeptor-Antagonisten MK-801 zu unterdrücken. Physiologisch scheint die Aktivierung des keratinozytären NMDA-Rezeptors mit der Differenzierung und insbesondere der orthologen Verhornung assoziiert zu sein. So konnte durch selektive Blockade des NMDA-Rezeptors mit MK-801 eine verminderte Expression der Differenzierungsmarker CK-10 und Filaggrin bei HaCaT-Zellen nachgewiesen werden [8,9]. Darüber hinaus korreliert die immunhistochemische Expression von NMDAR1 in der Epidermis mit der Expression des kalziumabhängigen Differenzierungsmarkers Filaggrin [9]. Dies ließ sich besonders für die parakeratotische Verhornung bei der Psoriasis vulgaris zeigen [9]. Im Gegensatz zu der Wertung, dass keratinozytäre NMDA-Rezeptoren für die kalziumabhängige Differenzierung bedeutsam sind, stellen Fuziwara et al. [11] einen negativen
Einfluss von NMDA-Rezeptoren auf die Barrierefunktion der Haut heraus, der durch eine gesteigerte Proliferation bedingt sein soll. Diese Argumentation fußt insbesondere auf der lokalen Applikation des selektiven Agonisten NMDA im Mausmodell, der zu einer verminderten Barrierefunktion führte. Mögliche zytotoxische, von der Rezeptorfunktion unabhängige Einflüsse (z.B. pH-Wert des aufgetragenen NMDA), blieben hierbei jedoch unberücksichtigt.

Außer den Daten zum NMDA-Rezeptor des Keratinozyten existieren nur wenige Arbeiten zur Präsenz anderer Glutamatrezeptoren. So wurden bei Ratten ionotrope Glutamatrezeptoren vom Typ des AMPA-Rezeptors immunhistochemisch in geringer Ausprägung im Stratum basale der Epidermis gefunden [12]. Untersuchungen zu keratinozytären Kainatrezeptoren wurden bislang nicht publiziert.

Für metabotrope Glutamatrezeptoren (mGluR) in Keratinozyten existieren bislang nur immunhistochemische Untersuchungen, die bei Ratten die Existenz von mGlu1 in der basalen Epidermis nachweisen konnten [12].

L-Glutamat ist der physiologische Transmitter der Glutamatrezeptoren. Es wird im Nervensystem von Gliazellen/Schwann'schen Zellen gebildet [17] oder von exzitatorisch wirkenden Neuronen in den synaptischen Spalt abgegeben. Im Vergleich zur Datenlage bei Nervenzellen sind die Erkenntnisse zu L-Glutamat als Transmitter bei Keratinozyten gering, weshalb derzeit vielfach noch Analogieschlüsse zum Nervengewebe notwendig sind. Im Unterschied zum Nervengewebe ist insbesondere die Herkunft des L-Glutamats, das am NMDA-Rezeptor des Keratinozyten bindet, unbekannt. Als möglicher Ursprung werden Schwann'sche Zellen, Makrophagen und Axone diskutiert [18]. Schwann'sche Scheidenzellen und Makrophagen sind jedoch als Quelle von epidermalem L-Glutamat allein aufgrund der Anatomie sehr unwahrscheinlich. Eine L-Glutamatversorgung von Keratinozyten durch intraepidermale Axone wäre hingegen grundsätzlich denkbar, da zumindest Axone der dermo-epidermalen Übergangszone sowohl L-Glutamat enthalten als auch zu dessen Biosynthese befähigt sind [19]. Möglich ist jedoch auch ein autokriner/parakriner Mechanismus, bei dem das am keratinozytären NMDA-Rezeptor wirkende L-Glutamat aus Keratinozyten stammt. Neuere Studien konnten eine Freisetzung von L-Glutamat durch kultivierte Keratinozyten (HaCaT) nachweisen [20]. Darüber hinaus ist Glutamat in der Epidermis mittels Immunfluoreszenz bzw. Immunhistochemie gefunden worden [11,21], wobei die Datenlage widersprüchlich ist. Während Fuziwara et al. [11] bei Nacktmäusen eine überwiegende Expression in der oberen Epidermis beschreiben, konnten Nordlind et al. [21] L-Glutamat in der gesamten Epidermis finden. Eine gleichmäßige Verteilung von L-Glutamat innerhalb der Epidermis würde allerdings die Vermutung nahe legen, dass außer dem NMDA-Rezeptor noch andere funktionell bedeutsame Glutamatrezepto- 
ren in der Epidermis vorhanden sind, sodass die biologische Funktion von L-Glutamat von der Verteilung der jeweiligen Rezeptorsubtypen in der Epidermis abhängt. Für diese These fanden sich in unserer Arbeitsgruppe zuletzt Hinweise, da bei kultivierten normalen humanen epidermalen Keratinozyten eine durch L-Glutamat ausgelöste Erhöhung der intrazellulären Kalziumkonzentration durch den selektiven NMDA-Rezeptorantagonisten AP5 nur partial antagonisiert werden konnte [22]. Welche Glutamatrezeptoren hierfür verantwortlich sind, ist bislang unbekannt.

Die bisherigen Erkenntnisse zu keratinozytären Glutamatrezeptoren zeigen, dass vermeintlich exklusiv neurogene Transmitter auch in der Epidermis spezifische und funktionell bedeutsame Effekte haben können. Interessanterweise ist dabei auch die Lokalisation des Rezeptorsubtyps für seine Funktion von Bedeutung und kann dazu führen, dass ein und derselbe Transmitter in Abhängigkeit der vorhandenen Rezeptorsubtypen und ihrer Lokalisation unterschiedliche, teils gegenläufige Wirkungen entfalten kann. Als Beispiel hierfür sind Pruinrezeptoren (Transmitter: ATP) zu nennen, die sowohl eine Förderung der Proliferation als auch der Differenzierung bewirken [3,5]. So ist der G-Protein-gekoppelte Subtyp P2Y2 des Purinrezeptors bei Keratinozyten im Stratum basale zu finden und führt nach Stimulation zur Proliferation, während P2X-Rezeptoren (Subtypen P2X5 und P2X7) in oberen Epidermisanteilen lokalisiert sind und calciumabhängig die Differenzierung von Keratinozyten fördern $[3,5]$. Ein ähnlicher Effekt ist bei Acetylcholinrezeptoren des Keratinozyten zu beobachten, die ebenfalls einen Einfluss auf die Proliferation und Differenzierung von Keratinozyten zeigen und innerhalb der Epidermis ein spezifisches Verteilungsmuster haben $[1,23]$. Auch hier haben die einzelnen Rezeptorsubtypen unterschiedliche Funktionen. So beeinflussen, abhängig vom jeweiligen Subtyp, muskarinische und nikotinische Acetylcholinrezeptoren die Proliferation und Differenzierung, aber auch die Migration und Adhäsion von Keratinozyten [23]. Diese Effekte werden bei den G-Protein-gekoppelten muskarinischen Acetylcholinrezeptoren durch Veränderungen im transmembranösen Kalziuminflux bewirkt, wohingegen die nikotinischen Acetylcholinrezeptoren verschiedene Ionenkanäle $\left(\mathrm{Na}^{+}, \mathrm{K}^{+}, \mathrm{Ca}^{++}\right)$öffnen können [23].

Die bislang vorliegenden Befunde zu ursprünglich neurogenen Transmittern und Rezeptoren bei Keratinozyten lassen vermuten, dass in der Epidermis ein ähnlich komplexes Signalnetzwerk vorhanden ist wie im Nervensystem. Dies bietet Ansatzpunkte für vielfältige Forschungsprojekte.

\section{Abstract}

\section{Transmitter Functions in Human Epidermis, Using Keratinocytic Glutamate Receptors as an Example $\nabla$}

Transmitters and receptors which were originally presumed to be located exclusively in the nervous system are increasingly being found in human epidermis. Among these are glutamate, acetylcoline and purin receptors. The individual receptor families influence the proliferation, differentiation of the keratinocytes as well as the barrier function of the epidermis. Using keratinocytic glutamate receptors, this article describes transmitter functions in the epidermis which were originally attributed solely to the nervous system.

\section{Literatur}

1 Kurzen H, Wessler I, Kirkpatrick CJ, Kawashima K, Grando SA. The nonneuronal cholinergic system of human skin. Horm Metab Res 2007; 39: $125-135$

2 Pullar CE, Rizzo A, Isseroff RR. beta-Adrenergic receptor antagonists accelerate skin wound healing: evidence for a catecholamine synthesis network in the epidermis. J Biol Chem 2006; 281: 21225 -21235

3 Greig AV, Linge C, Cambrey A, Burnstock G. Purinergic receptors are part of a signaling system for keratinocyte proliferation, differentiation, and apoptosis in human fetal epidermis. J Invest Dermatol 2003; 121: $1145-1149$

4 Grando SA, Pittelkow MR, Schallreuter KU. Adrenergic and cholinergic control in the biology of epidermis: physiological and clinical significance. J Invest Dermatol 2006; 126: 1948-1965

5 Inoue $K$, Hosoi J, Denda M. Extracellular ATP has stimulatory effects on the expression and release of IL- 6 via purinergic receptors in normal human epidermal keratinocytes. J Invest Dermatol 2007; 127: 362 371

6 Hardingham GE, Bading $H$. The Yin and Yang of NMDA receptor signalling. Trends Neurosci 2003; 26: $81-89$

$7 \mathrm{Kew} J N$, Kemp JA. Ionotropic and metabotropic glutamate receptor structure and pharmacology. Psychopharmacology 2005; 179: 4-29

8 Fischer M, Glanz D, William T, Klapperstück T, Wohlrab J, Marsch WC. $\mathrm{N}$-methyl-D-aspartate-receptors influence the intracellular calcium concentration of keratinocytes. Exp Dermatol 2004; 13: 512 -519

9 Fischer M, William T, Helmbold P, Wohlrab J, Marsch WC. Expression of epidermal N-methyl-D-aspartate receptors (NMDAR1) depends on formation of the granular layer-analysis in diseases with parakeratotic cornification. Arch Dermatol Res 2004; 296: 157- 162

10 Fischer M, Fiedler E, Seidel C, Glanz D, Helmbold P, Meiss F, Dammann R. Cultivated keratinocytes express N-methyl-D-aspartate-receptors of the NMDAR2D-type. Arch Dermatol Res 2006; 297: 316-318

11 Fuziwara S, Inoue $K$, Denda M. NMDA-type glutamate receptor is associated with cutaneous barrier homeostasis. J Invest Dermatol 2003; 120: $1023-1029$

12 Genever PG, Maxfield SJ, Kennovin GD, Maltman J, Bowgen CJ, Raxworthy MJ, Skerry TM. Evidence for a novel glutamate-mediated signaling pathway in keratinocytes. J Invest Dermatol 1999; 112: 337 - 342

13 Morhenn VB, Waleh NS, Mansbridge JN, Unson D, Zolotorev A, Cline P, Toll $L$. Evidence for an NMDA receptor subunit in human keratinocytes and rat cardiocytes. Eur J Pharmacol 1994; 268: 409-414

14 Morhenn VB, Murakami M, O'Grady T, Nordberg J, Gallo RL. Characterization of the expression and function of N-methyl-D-aspartate receptor in keratinocytes. Exp Dermatol 2004; 13: 505-511

15 Nahm WK, Philpot BD, Adams MM, Badiavas EV, Zhou LH, Butmarc J, Bear MF, Falanga V. Significance of N-methyl-D-Aspartate (NMDA) Receptor-mediated signalling in human keratinocytes. J Cell Physiol 2004; 200: 309-317

16 Menon GK, Elias PM. Ultrastructural localization of calcium in psoriatic and normal human epidermis. Arch Dermatol 1991; 127: 57-63

17 Wu SZ, Jiang S, Sims TJ, Barger SW. Schwann cells exhibit excitotoxicity consistent with release of NMDA receptor agonists. Neurosci Res 2005; 79: 638-643

18 Kinkelin I, Bröcker EB, Koltzenburg M, Carlton SM. Localization of ionotropic glutamate receptors in peripheral axons of human skin. Neurosci Lett 2000; 283: 149-152

19 Miller KE, Richards BA, Kriebel RM. Glutamine-, glutamine synthetase, glutamate dehydrogenase- and pyruvate carboxylase-immunoreactivities in the rat dorsal root ganglion and peripheral nerve. Brain Research 2002; 945: $202-211$

20 Hoogduijn MJ, Hitchcock IS, Smit NP, Gillbro JM, Schallreuter KU, Genever PG. Glutamate receptors on human melanocytes regulate the expression of MiTF. Pigment Cell Res 2006; 19: 58-67

21 Nordlind K, Johansson O, Liden S, Hokfelt T. Glutamate- and aspartatelike immunoreactivities in human normal and inflamed skin. Virchows Arch B Cell Pathol Incl Mol Pathol 1993; 64: 75-82

22 Fischer M, Glanz D, Presek P, Waltermann K, Brzoska T, Abels C. The effect of L-glutamate and L-aspartate on the intracellular calcium concentration of keratinocytes. Exp Dermatol 2007; 16: 273

23 Ndoye A, Buchli R, Greenberg B, Nguyen VT, Zia S, Rodriguez JG, Webber RJ, Lawry MA, Grando SA. Identification and mapping of keratinocyte muscarinic acetylcholine receptor subtypes in human epidermis. J Invest Dermatol 1998; 111: 410-416 